\title{
Constipation in childhood
}

\author{
W. HENDERSON \\ Consultant Paediatrician to the York Hospitals
}

THERE are numerous cases of alleged constipation in the early weeks and months of life which are simply examples of the normal but infrequent passage of stools in a contented breast fed baby. There are children who, throughout the 1st year of life are a little constipated because of an inappropriate diet-too much protein and too little sugar, too much of the solid constituents and too little fluid.

Constipation can be found in a secondary form in childhood, linked with fever, with the simple upset of having to stay in bed or of having to go to hospital, or with the more prolonged inconvenience of immobilization in splint or plaster.

There is a small number of medical conditions of which constipation may be quite an important part or occasionally a presenting symptom. To mention briefly only two of these we might consider cretinism and idiopathic hypercalcaemia.

Cretinism is not a very common condition but constipation is one of its characteristic features. Suspicion is usually aroused in the early months of the 1st year of life by delayed growth and slow mental activity and a characteristic facies. The skin feels dry, cold and rather thick. Diagnostic confirmation comes from radiological bone-growth assessment and biochemical investigation shows a low serum protein bound iodine level. Treatment with thyroid extract or thyroxine changes the clinical appearance and corrects the constipation.

Idiopathic infantile hypercalcaemia is usually a temporary disturbance (in its milder form) presenting at 5 or 6 months of age with failure to thrive, vomiting and often some irritability. Nearly always there is quite well marked constipation with hard pellet-like stools.

Biochemical investigation reveals a serum calcium level of over $12 \mathrm{mg} / 100 \mathrm{ml}$ and sometimes a raised level of blood urea. These infants are apparently sensitive to vitamin $D$, usually when it has been given in slight excess in the diet, but the mechanism of this aetiology is not quite clear. The clinical picture, including constipation, clears up on temporary withdrawal of vitamin $D$ from the diet.

For the rest of this talk I wish to consider constipation under the headings of organic and $\stackrel{\mathbb{Q}}{\varrho}$ functional. It is not misleading to suggest that is there is an age level dividing these two groups, $\vec{\circ}$ the organic cases occurring mostly before the age of 2 years, and the functional cases after the $\vec{\omega}$ age of 2 years. There is also a certain amount of truth in the simplification that the 'organic' group are constipated because they 'just can't' move their bowels and the functional group because they 'just won't'.

Organic group, this includes a variety of causes of which the most important are:

Congenital abnormalities of the anus.

Anal fissure.

Congenital aganglionosis of the large bowe or Hirschsprung's disease.

(1) Congenital abnormalities of the anal orific $\mathbb{B}$ and anal canal range from its complete absence (the rectum ending in a blind pouch) through \& $\mathbb{\mathbb { D }}$ group of malpositions, to the condition of cone $\vec{\theta}$ genital anal stenosis. The absence or malposition. of the anal orifice will be apparent at birth these cases are rare and require skilled investigation and surgical correction. Congenital anal stenosis is more common; it may present as frank constipation or may reveal itself by the passage of normal faeces in ribbon-like form as if squeezed from a tube. Dilation by bougies, repeated over a long period, is usually successful in overcoming the obstruction and allowing normal bowel function to develop.

(2) Anal fissure is very common and is still surprisingly often not diagnosed or not suspected as a cause of constipation in early childhood. The fissure or fissures can nearly always be seen on careful inspection. They usually follow a period in which the motions have been allowed to get hard and there is sometimes a history of blood being passed on the stool. This development produces anal spasm and painful defaecation. Local digital application of a cream or vaseline preparation containing a local anaesthetic is usually all that is required to bring about healing.

(3) Hirschsprung's disease. It is important to discuss this condition in some detail because of the importance of early diagnosis and because 
increasing numbers of infants are undergoing extensive surgery for its correction. The symptoms are due to the absence of parasympathetic ganglia in the submucous and intermuscular plexuses of the wall of the rectum and the lowest part of the sigmoid colon. In $30 \%$ of cases the aganglionic segment is in the rectum only, and in about $15 \%$ of cases it extends upwards beyond the sigmoid, even into the small intestine. The absence of ganglion cells interferes with the continuity of peristaltic activity and prevents relaxation in the affected part.

Difficulty in emptying the colon may be apparent within hours of birth, but occasionally is not suspected until some days, weeks or even months have passed. The proximal colon first dilates and then hypertrophies, producing the true megacolon; this may become sufficiently powerful to push the faecal matter through the narrow segment and prevent obstruction but sooner or later further help is required. This may take the form of 'medical' treatment by small regular rectal wash-outs, or by surgery-by colostomy or carefully planned corrective procedure.

In large series of cases it is clear that there is a wide range of severity in the preventing symptoms, some newborn infants are in need of colostomy on the 2nd or 3rd day of life, whilst some infants with intermittent obstructive symptoms are kept reasonably healthy by rectal wash-outs over a period of months.

It must be rare nowadays for a very mild case to escape detection until failure to grow adequately is noted to be linked with generalized gaseous distension of the abdomen and enquiry reveals a history of recurring bowel difficulties. But school age children are still met with in the out patient clinic who because of occasional constipation and a rather full abdomen have been referred with the suggestion that they might have Hirschsprung's disease.

The 'untreated' case of the years before surgery could help was a very miserable child with anorexia, stunted growth, and a distended tympanitic abdomen who suffered from chronic wasting and was likely to die from inhalational pneumonia or from acute entero-colitis.

Accurate diagnosis must always come before definitive surgery and there are several aspects of this :

The history when carefully taken may be very suggestive ;

X-Ray of the abdomen in the newborn period using only intestinal gas as the contrast medium may be very suggestive if it shows recognizable distension of the large bowel; but the more pre- cise definition of a narrow segment of rectum by the introduction of a small amount of opaque medium and careful positioning of the patient is now standard procedure.

Biopsy of the rectal mucosa and submucosa and if necessary of the intermuscular plexus area will usually provide a definite diagnosis. But satisfactory biopsy specimens are not always easy to obtain, nor do they show the extent of the aganglionic area.

Surgical correction of Hirschsprung's disease has been employed for just over 20 years. The original Swenson operation was a pull-through operation, an abdomino-perineal manoeuvre not usually carried out till the 2 nd year of life. There have been numerous subsequent modifications of the Swenson technique and new operations have been described in a number of widely separated surgical centres; it is clear that a completely satisfactory procedure has not yet been devised. The mortality rate in early surgery is still high and the sequelae of surgery include constipation, soiling and interference with pelvic nerve functions.

As surgery still provides the best chance of helping most cases the challenge to improve surgical techniques still remains.

So called functional constipation covers the conditions known as colonic inertia, better called rectal inertia, and that group referred to as functional megacolon but preferably labelled functional megarectum.

The picture has clarified a good deal in recent years and paediatricians with big departments and busy out patient clinics are very familiar with this problem.

The maximum age of presentation is probably about 6 years but ranges from 3 to 9 years. The age of onset of symptoms is always earlierabout age 2 . The condition is slightly more frequent in boys than in girls.

It is not uncommon for the child to present with faecal soiling or apparent faecal incontinence. In others the story is of chronic constipation, extending over long periods, usually of months; it is often reported that the child has not had its bowels opened for several days.

In many cases there is recurrent abdominal discomfort. There is no abdominal distension but large firm masses can be felt on palpation of the abdomen, occasionally they are so hard that they give rise to a suspicion of cancer. In these cases of functional constipation the normal reflex patterns of rectal behaviour have broken down and the rectum has got into the habit of relaxing to accommodate more and more faeces instead of being stimulated to evacuate. The active colon goes on pushing faeces into the rectum which 
distends and fills until a solid mass may be seen as well as felt to extend up to the rib margin ; in a few cases the distension of the large bowel extends proximally into the colon but for the most part it is the rectum which is involved. The colon remains functioning in a fairly normal fashion, intestinal gas can pass around and beyond the faecal accumulation so there is no gross distension of the abdomen. Some of the more liquid content of the colon can similarly pass the edges of the faecal mass and lead to faecal staining and leakage - the so called 'overflow incontinence'.

Rectal examination is absolutely essential in the clinical investigation of these cases; the rectum is invariably full of faeces which may be of firm or of hard consistency, and it is sometimes apparent that the anal orifice is patulous from straining.

In seeking a cause for this terminal state of rectal inertia several possibilities have to be borne in mind. A simple habit of infrequent evacuation may have been induced by illness, by hospitalization, or by repeated discomforts of a small anal fissure. But more often the starting point lies in that period of the child's development where 'bowel training' was being actively practised by the parents. Sometimes there has been too much emphasis placed by the parents on the need for regularity in obtaining bowel movement, or perhaps too strict a discipline imposed in an effort to get the infant 'clean' in its habits at an early age.

In other cases evacuation of the bowel has become too closely linked with the expression of emotion, a parent (usually the mother) hoping that the child will show its fondness and its love for her by 'obliging' her with the passage of a motion at an expected time. Any of these management situations involving over-emphasis may lead to the development of a mild resistance on the part of the child, and if the warning signs are not heeded or are wrongly interpreted than a fixed resistance to defaecation and deliberate 'holding back' is likely to follow.

It does not take long then for the rectum to become gradually over-distended and incapable of the ordinary contraction response. This state of affairs once developed tends to persist long after the initial causative situation has subsided. The development and persistence of the so called rectal inertia syndrome leads to considerable psychological upset in the family and often difficulties in attendance and performance at school.

Efforts have been made to define characteristic personality features in the parents of these children; they have been said to show obsessional rigidity or exaggerated perfectionism and one study of the background of parents suggested that they themselves had often been subjected to a similar strict code of training in infancy.

The clinical features of rectal inertia have been fully described and it remains only to say that the diagnosis is essentially made on the history and a careful clinical examination. There should seldom be any difficulty in separating this condition from Hirschsprung's disease and a helpful list of differentiating features is shown in Table 1 adapted from Nixon (1961).

The treatment of rectal inertia must be undero taken with firmness and assurance and aQ acceptance that it is a long term project. The worst case may require digital removal of hard faeces under general anaesthesia; a slightly leso 3 severe impaction may yield to gradual break $\subseteq$ down of the mass with a finger helpe $\vec{\theta}$ by repeated small (1-2 oz) rectal wash-outs wits. saline. Or rectal wash-outs using repeated smate amounts once a day may be repeated until the mass has been got rid of. Accumulations of softer consistence can of course be evacuated with much less trouble.

When the rectum has been satisfactorily emptied it is necessary to start a programme of 're-education' in an effort to re-establish the longlost reflex of contraction following filling.

This takes time and is best started by small enemas or wash-outs given twice weekly for 2 weeks then weekly for some further weeks according to need. This treatment must be simultaneously supported by regular laxatives-of which there is a wide choice. Suitable ones are

TABLE 1

\begin{tabular}{lcc}
\hline & Hirschsprung's disease & Rectal inertia \\
\hline Onset & Early-even neonatal & 'Training period' and later \\
Constipation & + & + \\
Distension & + & - \\
Peristalsis & ++ & - \\
Rectum & Empty & Loaded \\
Soiling & - & + \\
\hline
\end{tabular}

From Nixon (1961). 
Dulcolax, neostigmine mixtures and Senokotthis last can be given in the form of tablets or granules, the dosage is easily graded according to results and there are no side effects from prolonged administration.

The essence of treatment is that it should be thorough and long continued in view of the strong tendancy to relapse. A mild form of psychotherapy consisting of full explanation, reassurance, and continuing interest is a very necessary adjunct. Cases of long standing with a history of numerous relapses may require the help of a trained psychiatric team.
It seems possible that in a few cases the rectum never regains its basic contractility and that evacuation is eventually possible only with the help of the abdominal muscles.

Other local causes of hold up-for examples fissures-must of course be looked for and treated.

\section{Reference}

Nixon, H.H. (1961) Discussion on megacolon and megarectum. Proc. roy. Soc. Med. 54, 1037. 